\title{
Article
}

\section{Spectroscopically Determined Force Field for Water Dimer: Physically Enhanced Treatment of Hydrogen Bonding in Molecular Mechanics Energy Functions}

Berit Mannfors, Kim Palmo, and Samuel Krimm

J. Phys. Chem. A, 2008, 112 (49), 12667-12678 • Publication Date (Web): 14 November 2008

Downloaded from http://pubs.acs.org on December 4, 2008

\section{More About This Article}

Additional resources and features associated with this article are available within the HTML version:

- $\quad$ Supporting Information

- $\quad$ Access to high resolution figures

- $\quad$ Links to articles and content related to this article

- Copyright permission to reproduce figures and/or text from this article

View the Full Text HTML 


\title{
Spectroscopically Determined Force Field for Water Dimer: Physically Enhanced Treatment of Hydrogen Bonding in Molecular Mechanics Energy Functions
}

\author{
Berit Mannfors, Kim Palmo, ${ }^{\dagger}$ and Samuel Krimm* \\ LSA Biophysics, University of Michigan, 930 North University Avenue, Ann Arbor, Michigan 48109
}

Received: August 4, 2008; Revised Manuscript Received: October 3, 2008

Our ab initio transformed spectroscopically determined force field (SDFF) methodology emphasizes, in addition to accurate structure and energy performance, comparable prediction of vibrational properties in order to improve reproduction of interaction forces. It is now applied to the determination of a molecular mechanics (MM) force field for the water monomer and dimer as an initial step in developing a more physically based treatment of the hydrogen bonding that not only underlies condensed-phase water but also must be important in molecular-level protein-water interactions. Essential electrical components of the SDFF for monomer water are found to be the following: an off-plane charge distribution, this distribution consisting of four off-atom charge sites in traditional lone pair (LP) but also in inverted lone pair (ILP) positions; allowance for a diffuse size to these off-atom sites; and the incorporation of charge fluxes (i.e., the change in charge with change in internal coordinate). Parametrization of such an LP/ILP model together with the SDFF analytically transformed valence force field results in essentially exact agreement with ab initio (in this case MP2/6$31++G(d, p))$ structure, electrical, and vibrational properties. Although we demonstrate that the properties of this monomer electrical model together with its van der Waals and polarization interactions are transferable to the dimer, this is not sufficient in reproducing comparable dimer properties, most notably the huge increase in infrared intensity of a donor $\mathrm{OH}$ stretch mode. This deficiency, which can be eliminated by a large dipolederivative-determined change in the effective charge flux of the donor hydrogen-bonded $\mathrm{OH}$ bond, is not accounted for by the charge flux change in this bond due to the induction effects of the acceptor electric field alone, and can only be fully removed by an added bond flux associated with the extent of overlap of the wave functions of the two molecules. We show that this overlap charge flux (OCF) emulates an actual $\mathrm{O}-\mathrm{H} \cdots \mathrm{LP}-\mathrm{O}$ intermolecular dipole flux, reflecting the unitary nature of the hydrogen-bonded system in the context of MM-separable molecules. The effectiveness of incorporating the OCF noncanonical character demonstrates that a distinctively QM-unique property can be substantively represented in MM energy functions.

\section{Introduction}

In pursuing our goal of producing more physically accurate molecular mechanics (MM) force fields for biomacromolecules, we have paid particular attention to the importance of assuring consistent agreement with all elements of the classical physics of the system. Thus, we have required that, in addition to presently satisfactory structures and energies, interaction forces should be reproduced more accurately than is achieved in current standard force fields. Since what then follows is greater accuracy in reproducing vibrational properties, we call this a Spectroscopically Determined Force Field (SDFF). ${ }^{1}$ This ab initiotransformed energy function is based on the recognition that attention to vibrational properties, i.e., modes, frequencies, and intensities, ensures the accurate reproduction of interaction forces. ${ }^{2}$ Such attention to forces, whose correctness is not necessarily guaranteed by energy reproduction alone, ${ }^{3}$ is critical to achieving maximum accuracy in structure and molecular dynamics calculations.

The unique characteristic of the SDFF methodology is its use of an analytical transformation that automatically determines the valence parameters (i.e., the complete set of force constants and the intrinsic geometry parameters) from quantum mechanical (QM) equilibrium structures and Hessians in combination

\footnotetext{
* Corresponding author. Phone: (734)763-8081. E-mail: skrimm@ umich.edu.

$\dagger$ Present address: D. E. Shaw Research, LLC, New York, NY 10036.
}

with given nonbond potentials. ${ }^{4}$ This, of course, emphasizes the importance of assuring a comprehensive description of the nonbond terms, which we find also guarantees maximum transferability of the valence parameters. The QM calculations are also used to directly optimize other components of the force field. Since all QM quantities originate from the wave function and thus are internally self-consistent, the SDFF protocol also achieves this by using QM-derived polarizability (and derivatives) in combination with QM-derived charges (and dipole derivatives) to weave together a self-consistent electrical model. The SDFF methodology has been implemented to produce force fields for hydrocarbon chains of saturated, both linear, ${ }^{5}$ and branched, ${ }^{6}$ as well as olefinic ${ }^{7}$ types, for single chains as well as for crystals, ${ }^{8,9}$ and for peptide models such as $N$-methylacetamide $^{1}$ and an alanine dipeptide analogue, ${ }^{2}$ as well as providing the basis for calculating infrared (IR) intensities ${ }^{10}$ in addition to frequencies.

In view of its obvious importance in the computational simulations of biomolecular systems, we have initiated the development of an SDFF for water. Since the structure of this molecule is not at issue, the main concern in producing a reliable MM representation of water must be to achieve an accurate physical description of its interaction with other molecules. We begin with treatments of the monomer and dimer as an introduction to the study of peptide-bonding water molecules and larger water clusters. The SDFF approach in this case not 
only aims at the ultimate development of a comprehensive computational understanding of the properties of liquid water but also provides the opportunity to establish on a complete QM basis the physical requirements for a description of hydrogen bonding in the SDFF and more generally in MM force fields. Since we insist not only on a spectroscopically complete analysis of the intramolecular vibrations but also on that of the intermolecular modes, this methodology obviously provides a very sensitive probe of the hydrogen-bonding interactions.

In this paper we present the components that we have found to be important in such an SDFF for water. Our aim is to determine the kinds of physical terms in the energy function needed to provide (in addition to accurate structures and energies) maximally correct forces, and therefore vibrational dynamics, not simply to parametrize an arbitrary energy function to yield better frequencies. We wish to develop a transferable model that will finally account for condensed-phase properties on the basis of molecule-molecule interactions since we also want such an SDFF to be appropriate for specific protein-water interactions. As a start, by elucidating a deficiency in current energy function treatments of hydrogen bonding, our SDFF provides the most QM-compatible MM representation to date of this interaction in the water dimer.

\section{Calculations}

As in the case of our SDFF for macromolecules, ${ }^{1}$ we require that the SDFF for water reproduce a comprehensive set of QM properties. We have calculated these using the GAUSSIAN $98^{11}$ and GAUSSIAN $03^{12}$ programs mainly for structures and energies and the GAMESS ${ }^{13}$ program for electric potentials on planes. Although it is well-known that very high-level QM theory provides results in better agreement with experimental values, we have used the MP2/6-31++G(d,p) level of theory because it is the foundation of our present peptide-chain SDFF. ${ }^{1,2}$ In developing our models for biomolecules, we emphasize internal compatibility over using different levels for the various components. Since the SDFF protocol is strongly guided by overall QM agreement, we expect that, although specific parameter values of the energy function may change with the chosen basis set level, the primary physical features of our water model will not be altered.

Corrections, such as counterpoise ${ }^{14}(\mathrm{CP})$, for basis set superposition error (BSSE) due to limited basis functions should be included in QM calculations of clusters. Since it has been shown that without BSSE corrections clusters become too attractive ${ }^{15}$ we have applied CP corrections to dimer interaction energies in connection with the parametrization of our van der Waals (vdW) potential. Although not incorporated, our MP2/ $6-31++\mathrm{G}(\mathrm{d}, \mathrm{p})$ results show a $0.1 \AA$ elongation in the hydrogen bond distance of the optimized water dimer when the $\mathrm{CP}$ correction is taken into account, in comparison to $\mathrm{CP}$-uncorrected results, all $\mathrm{CP}$-corrected intermolecular vibrations come out with lower frequencies (e.g., $32 \mathrm{~cm}^{-1}$ in the hydrogen bond stretch vibration), and due to the small changes in the $\mathrm{OH}$ bond lengths the lowest $\mathrm{CP}$-corrected $\mathrm{OH}$ stretch frequency is calculated $18 \mathrm{~cm}^{-1}$ lower than that without $\mathrm{CP}$ corrections. Other studies have come to similar conclusions. ${ }^{16}$

The first step in our general SDFF procedure $^{1}$ is the parametrization of the nonbond terms, which starts with optimization of the electrical parameters mainly to ab initio electric potentials (since this can be done independently of other interactions, which do not contribute to the electric potential). As in our previous implementations, ${ }^{17,18}$ this was done by calculating the potential at points on carefully selected planes

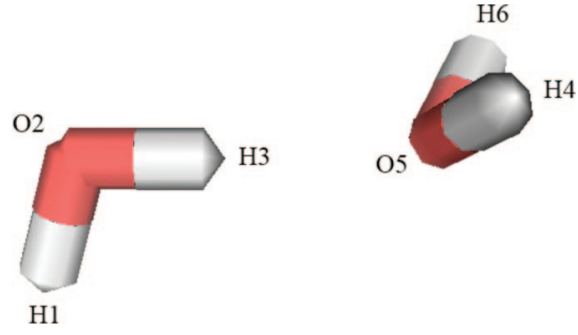

Figure 1. Sketch of $M P 2 / 6-31++G(d, p)$ water dimer with atom numbering.

through and outside the molecule. In the SDFF philosophy, monomer properties should be transferable to clusters, with cluster-specific physical features being additionally included as required. Thus, we first optimize the parameters for the water monomer, which in this case encompassed potentials on a plane containing the molecule, planes perpendicular to the molecular plane and containing the $\mathrm{OH}$ bond and the bisector of the $\mathrm{HOH}$ angle, and planes perpendicular to the molecular plane and perpendicular to the bisector. Electron density profiles around the monomer were also obtained, on planes through and outside the molecule. Such potentials and electron densities were also calculated in the presence of homogeneous electric fields of varying strengths and directions with respect to the molecule.

After electrical parameters are determined, our SDFF proto$\mathrm{col}^{1}$ proceeds to the determination of the vdW parameters, in this case by fitting to ab initio (CP-corrected) interaction energies of multiple configurations of fixed-monomer dimers (in order to minimize geometry-related effects) at several intermonomer distances in the range of 1.8 to $5.0 \AA$ (in some cases up to 15 $\AA$ ). Such configurations included $\mathrm{H}_{2} \mathrm{O} \cdots \mathrm{OH}_{2}$ approaches along the bisectors with molecular planes parallel and perpendicular; $\mathrm{OH}_{2} \cdots \mathrm{H}_{2} \mathrm{O}$ approaches of similar kinds; stacked approaches with molecular planes parallel and dipole moments parallel, antiparallel, and perpendicular; as well as analogues of the hydrogen-bonded dimer structure.

\section{Physical Features and Parameters of SDFF Water}

As the results of $\mathrm{QM}$ calculations on the water monomer $\left(\mathrm{H}_{1}-\mathrm{O}_{2}-\mathrm{H}_{3}\right)$ and water dimer $\left(\mathrm{H}_{1}-\mathrm{O}_{2}-\mathrm{H}_{3} \cdots \mathrm{O}_{5} \mathrm{H}_{4,6}\right.$, Figure 1) were assimilated and compared to various essential characteristics of the model, it became clear that certain requirements had to be incorporated into an SDFF model of water. It is important to note that no changes are made to the basic form of the SDFF energy function, ${ }^{1}$ which, designed to reproduce the Born-Oppenheimer surface, incorporates (quadratic, cubic, and quartic) diagonal and (quadratic) off-diagonal valence terms, Fourier-series torsions, a Lennard-Jones (L-J) type of vdW term, charge and dipole electrical interactions that include fluxes as well as static components, and polarization to account for induced effects. Only the values and physical meanings of specific parameters are affected by the hydrogen-bonding interaction. We first consider the elements required in an isolated water monomer, and through our studies of the monomer in an electric field environment (as is found in the dimer) we move to an investigation of the interactions in the actual water dimer. In this sequential way we have been able to determine some of the important physical features involved in hydrogen-bonded water-water interactions and to suggest the ways in which other MM functions may need to be modified to account for dimer properties.

3.1. Essential Components of Monomer SDFF Water. Since there are no intramolecular (1,4 and higher) vdW or electrostatic interactions in water, the SDFF transformation ${ }^{4}$ of 


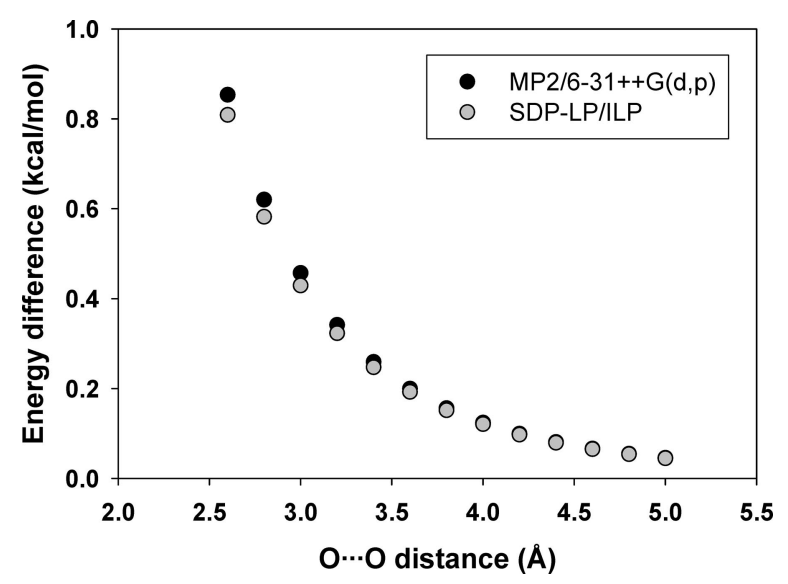

Figure 2. Interaction energy difference (in $\mathrm{kcal} / \mathrm{mol}$ ) between coplanar and perpendicular MP2/6-31++G(d,p) fixed-monomer $\mathrm{H}_{2} \mathrm{O} \cdots \mathrm{OH}_{2}$ configurations as a function of $\mathrm{O} \cdots \mathrm{O}$ distance (in $\AA$ ) at the MP2/6$31++\mathrm{G}(\mathrm{d}, \mathrm{p})$ level (black circles) and with our SDP-LP/ILP model (gray circles).

the monomer gives the valence force constants and the intrinsic structure parameters equal to the ab initio values. A detailed study of various aspects of such a model has yielded the following features.

3.1.1. Off-Plane Charge Distribution. In examining the various force field representations for the electrical interactions, we have also found, as has long been known, that a simple nucleus-centered point net charge model gives a bad reproduction of the electric potentials ( $r r m s(V)=14.9 \%$; see Table S1 of the Supporting Information). Although a significant improvement can be achieved by adding a (point) dipole at the $\mathrm{O}$ nucleus (rrms $(V)=4.9 \%$, Table S1 of the Supporting Information and ref 17), all planar component arrangements still result in a zero perpendicular quadrupole moment element $\left(Q_{z z}\right.$ in Table $\mathrm{S} 1$ of the Supporting Information) in the nonzero-trace representation (the ab initio value being nonzero), which can only be corrected by some off-atom charge distribution or by explicitly designed electrical multipoles. Interestingly, additional evidence for a nonplanar charge distribution comes from the nonzero energy difference between the coplanar and perpendicular plane arrangements of the fixed-monomer $\mathrm{H}_{2} \mathrm{O} \cdots \mathrm{OH}_{2}$ dimer configurations. At an $\mathrm{O} \cdots \mathrm{O}$ separation of $2.6 \AA$ this MP2 energy difference is $0.85 \mathrm{kcal} / \mathrm{mol}$, and for any reasonable potentialderived atomic point charge model the electrostatic difference due to the $\mathrm{H}$ atoms alone is $\sim 0.1 \mathrm{kcal} / \mathrm{mol}$ for a $\sim 0.4 e \mathrm{H}$ charge. Since the cylindrically symmetric electric fields of these configurations lead to a zero contribution from induction energies, and the total vdW contribution (as well as separate $\mathrm{H} \cdots \mathrm{H}, \mathrm{O} \cdots \mathrm{O}$, and $\mathrm{H} \cdots \mathrm{O}$ contributions) is essentially zero, it follows that the $\sim 0.7 \mathrm{kcal} / \mathrm{mol}$ remainder must be attributable to nonplanar electrostatic interactions. This is also strongly supported by the slower-than-vdW falloff in energy with $\mathrm{O} \cdots \mathrm{O}$ separation, $\sim r^{-5}$, as shown in Figure 2 .

3.1.2. Off-Atom Charge Sites. Granted that there is no unique way for a model to represent with accuracy and convergence the electric potential around a molecule, nevertheless a choice designed to achieve "chemical reasonableness and transferability" can be effective. ${ }^{19}$ Our determined essentially spherical electron density around the $\mathrm{O}$ nucleus as well as its gas-like behavior when placed in a homogeneous electric field (i.e., slightly deformed but with no visible lone pair (LP) characteristics) suggest a spherical off-atom arrangement of charges. However, on planes beyond $\sim 0.5 \AA$ outside the molecule near the $\mathrm{O}$ nucleus and perpendicular to the bisector of the $\mathrm{HOH}$
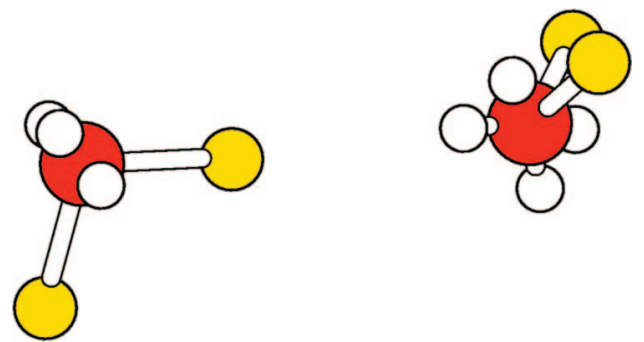

Figure 3. SDP-LP/ILP water dimer: oxygen, red; hydrogen, yellow; off-atom sites, white.

angle the electron density pattern is distinctly elliptical, with an indication of extension in the traditional lone pair directions (i.e., in the plane through the bisector and perpendicular to the plane of the molecule). We have chosen to represent this external anisotropy, as have others ${ }^{20,21}$ and is supported by electron localization function studies, ${ }^{22}$ by LP off-atom charges attached to the $\mathrm{O}$ nucleus. Aside from the obvious nonsphericity of such an LP-only model, calculated electron density profiles indicated that the electron cloud around the $\mathrm{O}$ nucleus is also shifted toward the $\mathrm{H}$ nuclei, and the optimization to the QM electric potential additionally pointed to the necessity of adding two off-plane charges attached to the $\mathrm{O}$ nucleus and in the plane of the LP charges (the bisector plane) but pointing toward the $\mathrm{H}$ nuclei. We call these an inverted lone pair (ILP), and note that a single such configuration was indicated by earlier studies aimed at finding the optimal location of water-oxygen lone pair charge sites. ${ }^{23}$ Our combined set of four off-atom charges with the traditional three charge sites at the positions of the nuclei, which we call a lone pair/inverted lone pair (LP/ILP) model, is the simplest off-atom charge arrangement to provide a more accurate reproduction of our selected QM properties. Of course, the off-atom charge parameters (charge, distance from the $\mathrm{O}$ nucleus, and location with respect to the bisector of the $\mathrm{HOH}$ angle) need to be determined by optimization to appropriate ab initio properties. In the course of studying such optimizations, we also examined additional dispersion of these charges but did not find that this results in any significant improvement in properties.

Such off-atom sites, illustrated in Figure 3 for the dimer, are incorporated in the SDFF in the following manner. Any number of off-atom charge sites may be positioned relative to a host atom and a few reference atoms in the same covalent structure. Since we use bond charge increments (bci), every off-atom site also has a counterion that is placed on the host atom. At each host atom, a molecule-fixed coordinate frame is constructed based on the given configuration of reference atoms. For water, the oxygen is chosen as host atom, and the local axes consist of three vectors (if off-plane sites are used), i.e.,

$$
\begin{aligned}
& \mathbf{v}_{1}=N_{1}\left(\mathbf{e}_{r 1}+\mathbf{e}_{r 2}\right) \\
& \mathbf{v}_{2}=N_{2}\left(\mathbf{e}_{r 1}-\mathbf{e}_{r 2}\right) \\
& \mathbf{v}_{3}=N_{3}\left(\mathbf{e}_{r 1} \times \mathbf{e}_{r 2}\right)
\end{aligned}
$$

where $\mathbf{e}_{r 1}$ and $\mathbf{e}_{r 2}$ are bond unit vectors going out from the oxygen, and $N_{1}, N_{2}$, and $N_{3}$ are "normalization" factors. The normalization is done once, at a chosen reference geometry, and $N_{1}, N_{2}$, and $N_{3}$ are held fixed thereafter. The distance vector $\mathbf{d}_{i}$ of an off-atom charge site $i$ from its host atom is then determined by three coefficients $c_{i}$,

$$
\mathbf{d}_{i}=c_{i 1} \mathbf{v}_{1}+c_{i 2} \mathbf{v}_{2}+c_{i 3} \mathbf{v}_{3}
$$

The vectors $\mathbf{v}_{1}, \mathbf{v}_{2}$, and $\mathbf{v}_{3}$ are not unit vectors at all geometries, which has the intended consequence of making the off-atom 
sites obey the local symmetry. For example, if the $\mathrm{HOH}$ angle becomes linear, all these sites in a plane perpendicular to the molecule, but containing the bisector, will coincide with the oxygen (i.e., with their counterions) and therefore be neutralized. Off-atom charge sites on the same host atom do not interact with each other or with the host atom. This also holds for offatom charges on different host atoms if the respective host atoms are excluded from electric interactions, such as atoms in the 1,2 and 1,3 positions.

3.1.3. Diffuse Off-Atom Charges. In view of the diffuse character of the electron density, we have examined whether it would be useful to give some extension to the charge distribution of a water monomer by allowing for diffuse off-atom sites in our SDFF, i.e., by giving a "size" to the off-atom charges. A useful technique for doing this, originating from fluid dynamics, ${ }^{24,25}$ is to replace the distance $R$ from the charge to a point by the buffered distance

$$
R_{s}=\sqrt{R^{2}+s^{2}}
$$

where $s$ is the buffer size. At close distances, this makes the charge behave like a charge distribution, ${ }^{26}$ with almost no computational overhead. Actually, the only additional computation required is to replace $R$ by $R_{s}$. There are no additional terms in the derivatives with respect to the Cartesian coordinates. This is seen in the following way. Since $R_{s}{ }^{2}=\mathbf{R} \cdot \mathbf{R}+s^{2}$, we have

$$
R_{s} \frac{\partial R_{s}}{\partial x_{k \alpha}}=\mathbf{R} \cdot \frac{\partial \mathbf{R}}{\partial x_{k \alpha}}
$$

so that

$$
\frac{\partial R_{s}}{\partial x_{k \alpha}}=\frac{\mathbf{R}}{R_{s}} \cdot \frac{\partial \mathbf{R}}{\partial x_{k \alpha}}
$$

This expression contains the same terms for all values of $s$, including $s=0$. It is straightforward to show that the second (and higher) derivatives also do not contain any additional terms for nonzero $s$. Note that $R_{s}$ is a scalar only; the vector $\mathbf{R}$ remains unchanged and does not depend on $s$.

3.1.4. Incorporation of Charge Flux. As noted above, an important feature of the SDFF (not included in currently standard MM functions) is the incorporation of charge fluxes, i.e., change in charge with change in internal coordinate. For a bci, $q(b)$, associated with a bond $b$ we have ${ }^{1}$

$$
q(b)=q_{0}(b)+\sum_{j} a_{b j}\left(S_{j}-S_{j 0}\right)
$$

where $q_{0}(b)$ is the bci at zero deformation of the internal coordinates $S_{j}$, and $a_{b j}$ is the charge flux, i.e.

$$
a_{b j}=\frac{\partial q(b)}{\partial S_{j}}
$$

For water $\mathrm{OH}$ bonds we define $q(b)$ as the charge on the $\mathrm{H}$ atom, the counter-charge, $-q(b)$, being added to the $\mathrm{O}$ atom. The most significant charge flux in the context of this work turns out to be the diagonal $\mathrm{OH}$ bond charge flux, i.e., the rate at which the $\mathrm{OH}$ bci changes when that same bond is elongated. As is well-known ${ }^{27,28}$ and has been further emphasized, ${ }^{29}$ such charge fluxes, which are obtained directly by optimization to ab initio dipole derivatives, are intrinsic properties of the isolated molecule in the absence of electric fields (and in the case of the water monomer, of internal polarization interactions), lead to the coupling of intramolecular and intermolecular coordinates, and are involved in providing correct forces, both internal as well as in interactions with other molecules. ${ }^{2}$ We have empha- sized that they should therefore be included in MM energy functions independent of polarization to provide needed physical accuracy. ${ }^{2}$ We have also shown that charge fluxes in the SDFF serve not only to predict correct IR intensities ${ }^{10}$ but to understand structural changes such as nitrogen pyramidalization in peptides $^{30}$ and the electrical contribution to peptide torsion potentials. $^{2}$ It is therefore not surprising that such fluxes are particularly important in an MM function for water, where, as we have already shown, ${ }^{2}$ they play the central role in accounting for the $\mathrm{HOH}$ angle increase from monomer to dimer and the liquid state as well as the variation of this angle with temperature.

3.1.5. Polarization. Polarization can be an important component in describing electrical interactions in MM energy functions, ${ }^{31}$ although it has been noted that the charge fluxes can have a comparable or even larger effect. ${ }^{28,32}$ While it is only applicable in the presence of external electrical interactions, as in the dimer, we include polarization here as a property of the monomer. We have implemented linear polarization in our SDFF, using our recently developed group polarizability model for water, ${ }^{33}$ since it may be important in clusters and in the condensed phase. It also improves the agreement in the third lowest intermolecular frequency and intensity of the dimer.

The complete set of the final electrical and force constant parameters of the water monomer is given in Table 1. The corresponding parameters for various nonpolarizable test models, as well as the monomer and dimer electrical, structural, and vibrational properties resulting from them, are given in the Supporting Information (Tables S1 and S2). Although the vdW interactions occur between monomers (and are discussed in that connection below), they represent properties of the monomer and their parameters are therefore included in Table 1. The combination of the parameters of the electrical model and the $\mathrm{vdW}$ interactions together with the SDFF-transformed valence force field constitute our monomer SDFF water, which we term a Spectroscopically Determined Polarizable Lone Pair/Inverted Lone Pair (SDP-LP/ILP) model.

3.2. Additional Components of Dimer SDFF Water and Physical Features for Treating Hydrogen Bonding. In the spirit of the standard formulation of MM energy functions, we expect dimer SDFF water to incorporate the basic monomer parameters, such as the intrinsic structural parameters and force constants as well as the electrical model (see Table 1). The obviously needed additional components would involve the nonbond terms such as the vdW interactions and the effects of polarization (already incorporated in Table 1; see section 3.1.5). The hydrogen bond, however, clearly may need special treatment since the close approach of donor and acceptor introduces nonclassical effects that are the result of the overlapping of the wave functions of the two molecules. ${ }^{34}$ To assess this possibility, we explore the induction contributions by determining the effects of the electric field of the acceptor on the properties of the donor and then assessing the extent to which the implementation of conventional MM energy terms may need to be adjusted.

3.2.1. van der Waals Potential. The optimization of $\mathrm{vdW}$ parameters is in general nontrivial and nonunique, ${ }^{1}$ but we found that although this was true for nonrealistic electrical water models the parameters became unique for more realistic models. We used the $m-n$ L-J vdW potential in the A-B form ${ }^{5}$ since this permits separate evaluations of the repulsive and attractive parameters. Optimization of the A and B parameters to our fixed-monomer dimer configurations revealed two important features. First, use of a 12-6 potential did not allow a reasonable optimization of attractive parameters, and various properties 
TABLE 1: SDF-LP/ILP Water Monomer Parameters and Dimer-Specific Charge Fluxes

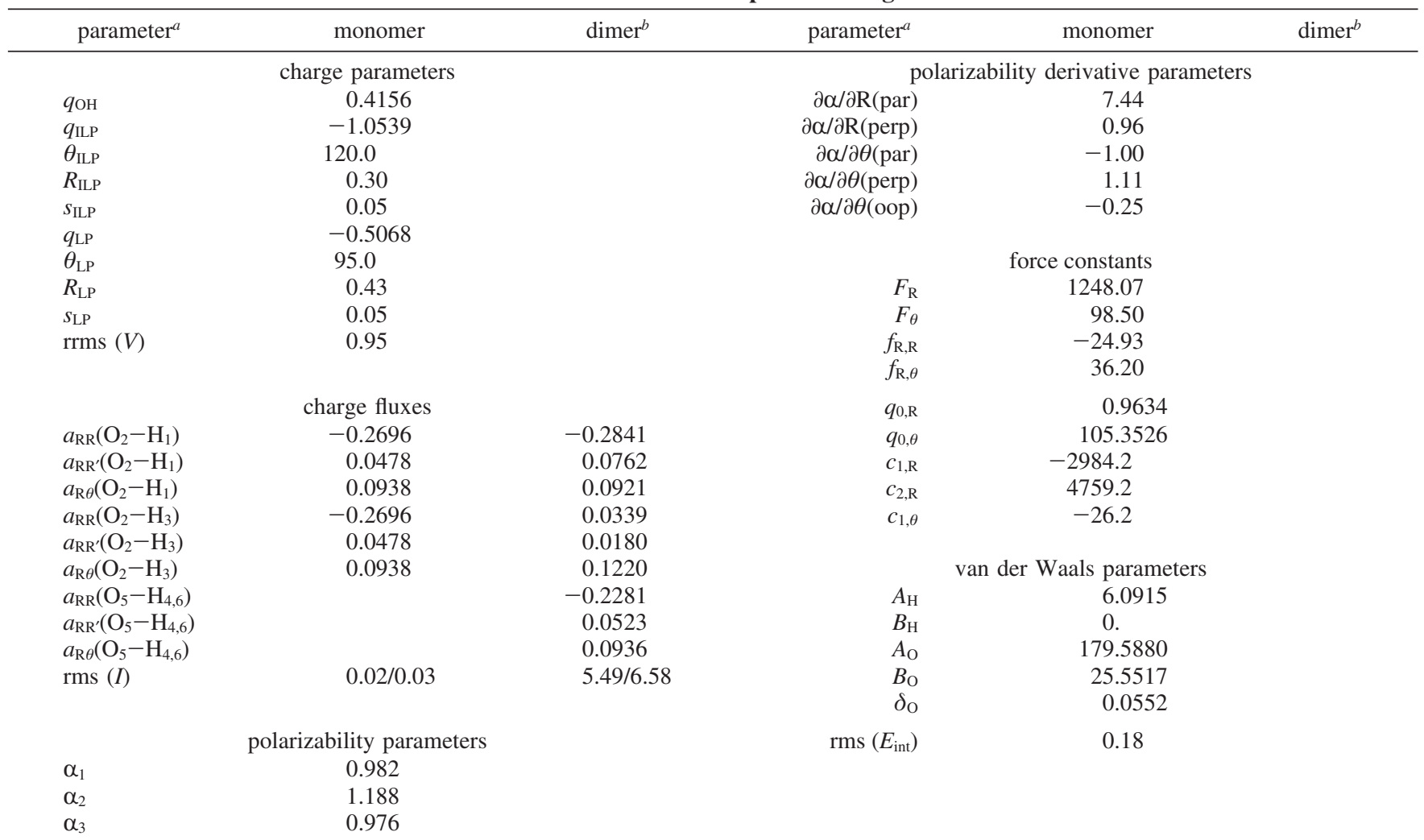

${ }^{a}$ The notation and the units for the parameters are the following: (1) Electric model optimized for the ab initio energy-minimized geometry to the ab initio electric potentials: $q_{\mathrm{OH}}$ is the $\mathrm{OH}$ bond charge increment (i.e., the charge of the $\mathrm{H}$ atom in electrons); $q_{\mathrm{ILP}}, \theta_{\mathrm{ILP}}, R_{\mathrm{ILP}}$, and $s_{\mathrm{ILP}}$ are respectively the charge at the off-atom ILP site (in electrons), the angle between the ILP charge sites (in deg), the distance between the oxygen nucleus and the ILP charge site (in $\AA$ ), and the size of the ILP charge (in $\AA$ ); $q_{\mathrm{LP}}, \theta_{\mathrm{LP}}, R_{\mathrm{LP}}$, and $s_{\mathrm{LP}}$ are the corresponding parameters of the off-atom LP charge sites; and rrms $(V)$ is the relative root-mean-square deviation (in \%) from the MP2/6-31++G(d,p) electric potentials. (2) Charge fluxes optimized to the ab initio dipole derivatives: $a_{\mathrm{RR}}$ is the diagonal OH bond charge flux parameter (in $e / \AA$ ); $a_{\mathrm{RR}}$ (in $e / \AA$ ) and $a_{\mathrm{R} \theta}$ (in $e / \mathrm{rad}$ ) are the $\mathrm{OH}$ bond charge flux cross terms due to the deformation of the other $\mathrm{OH}$ bond and the $\mathrm{HOH}$ angle, respectively; and rms (I) is the root-mean-square deviation (in $\mathrm{km} / \mathrm{mol}$ ) from the MP2/6-31++G(d,p) dipole derivative-determined IR intensities. The first rms deviation is for all and the second for all non-OH stretch vibrations (including intermolecular vibrations). (3) Polarizability parameters optimized to the ab initio electric potentials in the presence of external homogeneous electric fields: $\alpha_{1}, \alpha_{2}$, and $\alpha_{3}$ are respectively the group polarizability parameters (in $\AA^{3}$ ) along the bisector of the $\mathrm{HOH}$ angle, perpendicular to the bisector but in the molecular plane, and perpendicular to the molecular plane. These are located on the bisector, $0.19 \AA$ from O toward the $\mathrm{H}$ atoms (ref 33). (Polarizability does not affect the calculation of the isolated water monomer properties.) (4) Polarizability derivatives (in atomic units) are directly derived from QM (here MP2/6-31++G(d,p)): $\partial \alpha / \partial \mathrm{R}($ par) and $\partial \alpha / \partial \mathrm{R}($ perp) are the polarizability derivatives with respect to the $\mathrm{OH}$ bond (par $=$ parallel to the bond, perp $=$ perpendicular to the bond); $\partial \alpha / \partial \theta$ (par), $\partial \alpha / \partial \theta$ (perp), and $\partial \alpha / \partial \theta($ oop) are the polarizability derivatives with respect to the HOH angle $($ par $=$ along the bisector, perp $=$ perpendicular to the bisector but in the molecular plane, oop $=$ perpendicular to the molecular plane). (Polarizability derivatives do not affect the calculation of the isolated water monomer properties.) (5) Valence force constants (for the functional form, see ref 1): $F, f, q_{0}, c_{1}$, and $c_{2}$ are respectively the diagonal force constant, the interaction force constant (cross-term), the intrinsic structure parameter, and the cubic and quartic anharmonicity parameters. $R$ refers to $\mathrm{OH}$ stretch and $\theta$ to $\mathrm{HOH}$ angle bending. The energy is in $\mathrm{kcal} / \mathrm{mol}$, the bond length in $\AA$, and the angle in radians. (6) van der Waals parameters (for the functional form, see ref 1 ): $A_{\mathrm{H}}$ and $B_{\mathrm{H}}$ are the repulsive and attractive parameters of the hydrogen atom, respectively; $A_{\mathrm{O}}$ and $B_{\mathrm{O}}$ are the repulsive and attractive parameters of the oxygen atom, respectively; $\delta_{\mathrm{O}}$ is the shift parameter of the repulsive interaction center (in $\AA$ ) of the oxygen atom; and rms ( $E_{\text {int }}$ ) is the root-mean-square deviation (in $\mathrm{kcal} / \mathrm{mol}$ ) from the $\mathrm{MP} 2 / 6-31++\mathrm{G}(\mathrm{d}, \mathrm{p}) \mathrm{CP}$-corrected interaction energies. The energy is in $\mathrm{kcal} / \mathrm{mol}$ and the distance in $\AA .{ }^{b}$ All parameters optimized to the monomer except the charge fluxes are transferred directly to the dimer for the dimer SDFF.

improved as $m$ decreased to the 9 to 7 range. Since we found the 9-6 potential to be very satisfactory for the hydrocarbon SDFFs,${ }^{5}$ we retained this form for the L-J potential. Second, close contact repulsive interaction energies of the dimer configurations, especially those of the $\mathrm{O} \cdots \mathrm{O}$ configurations, were consistently larger than the corresponding ab initio values, suggesting that the optimal location of the interaction center for the repulsive vdW term of the $\mathrm{O}$ atom might not be the nucleus itself. Indeed, optimization corrected this over-repulsion in the dimer interaction energies and placed this interaction center $\boldsymbol{\delta}=\delta_{\mathrm{O}}\left(\mathbf{O}_{2} \mathbf{H}_{1}+\mathbf{O}_{2} \mathbf{H}_{3}\right)=0.064 \AA$ from the $\mathrm{O}$ nucleus along the bisector toward the $\mathrm{H}$ nuclei (where $\delta_{\mathrm{O}}$ is the shift parameter, given in Table 1 , and $\mathbf{O}_{2} \mathbf{H}_{1}$ and $\mathbf{O}_{2} \mathbf{H}_{3}$ are bond vectors). This again reflects the fact that the electron cloud around the $\mathrm{O}$ nucleus is shifted toward the $\mathrm{H}$ atoms, and in a sense is equivalent to providing some anisotropy to the vdW interaction of the $\mathrm{O}$ atom. Interestingly, roughly this shift is also given by the independently determined parameters of the LP and ILP sites. Similar optimizations for the H atom had only minor effects on dimer energetics, and the vdW interaction center of this atom was left at the nucleus. Our studies showed that the attractive parameter of the $\mathrm{H}$ atom is very small and poorly determined, and we have therefore constrained it to zero (see Table 1 for the optimized vdW parameters). The inclusion of the optimized shift for the interaction center of the $\mathrm{O}$ repulsive $\mathrm{vdW}$ term also resulted in better agreement between the SDFF 
TABLE 2: Properties of the SDP-LP/ILP Water Monomer ${ }^{a}$

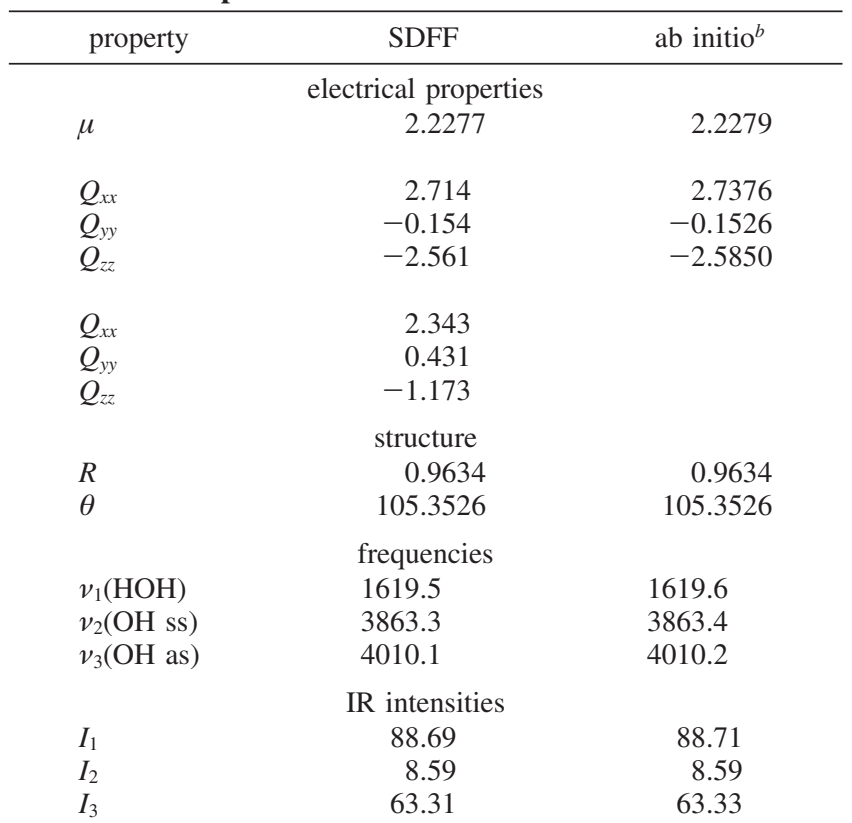

${ }^{a}$ The notation and the units for the properties are the following: (1) Electrical properties: $\mu$ is the molecular dipole moment (in debyes); and $Q_{x x}, Q_{y y}$, and $Q_{z z}$ are the diagonal quadrupole moment elements (in Buckinghams, or debye $\AA$ ), the first and second sets being the components of the traceless matrix and the matrix with a non-zero trace, respectively. The molecule is in the $x y$ plane and its dipole moment is along the $y$ axis. (2) Structural and vibrational properties: $R$ is the $\mathrm{OH}$ bond length (in $\AA$ ); $\theta$ is the $\mathrm{HOH}$ valence angle (in deg); $v_{1}(\mathrm{HOH}), v_{2}(\mathrm{OH} \mathrm{ss})$, and $v_{3}(\mathrm{OH}$ as) are respectively the $\mathrm{HOH}$ angle bending, symmetric $\mathrm{OH}$ stretch, and antisymmetric $\mathrm{OH}$ stretch frequencies (in $\mathrm{cm}^{-1}$ ); and $I_{1}, I_{2}$, and $I_{3}$ are the IR intensities (in $\mathrm{km} / \mathrm{mol}$ ) of the particular intramolecular vibrations. ${ }^{b} \mathrm{MP} 2 / 6-31++\mathrm{G}(\mathrm{d}, \mathrm{p})$.

and ab initio interaction energies (the rms deviation decreased from $\sim 0.4$ to $\sim 0.2 \mathrm{kcal} / \mathrm{mol}$ ) and improvements in the reproduction of the two lowest intermolecular frequencies.

3.2.2. Hydrogen Bonding. As expected, it is clearly evident that using only intrinsic monomer parameters in the water dimer does not correctly describe the physics that is essential in the hydrogen bonding interaction: force agreement, which would produce mode, frequency, and intensity agreement, ${ }^{2}$ is unacceptably poor. A particular indication of this is seen in the apparent large change in the optimized diagonal $\mathrm{O}_{2}-\mathrm{H}_{3}$ bond charge flux from the monomer value of $-0.2696 e \AA^{-1}$ (Table 1) to the effective (to be defined below) dimer value of +0.0339 $e \AA^{-1}$ (see Table 1). This change is required to obtain acceptable reproduction of the experimentally well-known dramatic IR intensity increase in a donor hydrogen-bonded $\mathrm{OH}$ stretch mode compared to the isolated monomer $\mathrm{OH}$ stretch mode (from an ab initio monomer value of $8.59 \mathrm{~km} / \mathrm{mol}, I_{2}$ in Table 2 , to a dimer value of $261.7 \mathrm{~km} / \mathrm{mol}, I_{9}$ in Table 3 ). This change indicates, as is well-known, ${ }^{22}$ that an important electronic reorganization is taking place in the dimer system. In the spirit of our MM philosophy, we want to incorporate in our SDFF the physical basis for such changes, and we began by exploring the induction effects of the electric field of the acceptor on the donor, electrical interactions being a key ingredient in hydrogen bonding. ${ }^{35}$ It should be noted that the electric field referred to here is that due to the classical field of the acceptor MM charge distribution and is not to be construed as associated with the full electron distribution in the hydrogen-bonded system. In the MM representation, any effects due to QM wave function overlap would have to be treated independently.
TABLE 3: Properties of the SDF-LP/ILP Water Dimers ${ }^{a}$

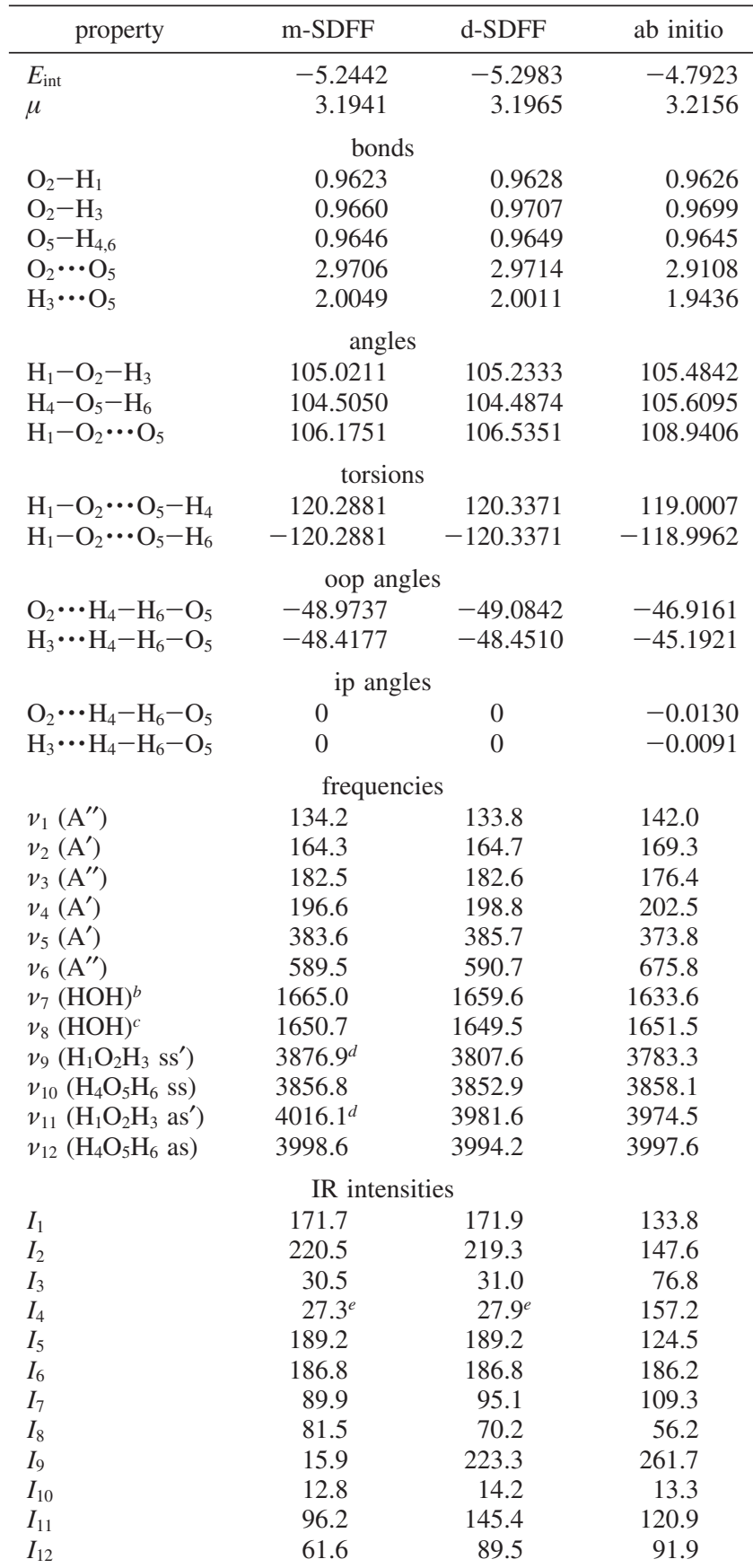

${ }^{a}$ The notation and the units for the properties are the following: $\mathrm{m}$-SDFF is the SDP-LP/ILP model (i.e., with monomer charge fluxes), and d-SDFF is the SDP-LP/ILP with individually optimized dimer charge fluxes. (1) $E_{\mathrm{int}}$ is the interaction energy in $\mathrm{kcal} / \mathrm{mol}$, and $\mu$ is the molecular dipole moment in debyes. (2) Structural properties: bond length is in $\AA$ and angle (valence, torsion, out-of-plane and in-plane) in deg. In this case we use dihedral angle for torsion, Wilson's definition [Wilson, E. B.; Decius, J. C.; Cross, P. C. Molecular Vibrations. The Theory of Infrared and Raman Vibrational Spectra; McGraw-Hill: New York, 1955] for out-of-plane angle, and an average of the difference of the valence angles around the bond $\mathrm{O}_{2} \cdots \mathrm{O}_{5}$ or $\mathrm{H}_{3} \cdots \mathrm{O}_{5}$ (i.e., $1 / 2\left(\mathrm{O}_{2} \cdots \mathrm{O}_{5}-\mathrm{H}_{4}-\mathrm{O}_{2} \cdots \mathrm{O}_{5}-\mathrm{H}_{6}\right)$ or $\left.{ }^{1} / 2\left(\mathrm{H}_{3} \cdots \mathrm{O}_{5}-\mathrm{H}_{4}-\mathrm{H}_{3} \cdots \mathrm{O}_{5}-\mathrm{H}_{6}\right)\right)$ for in-plane angle. (3) Frequency $v$ is in $\mathrm{cm}^{-1}$, IR intensity $I$ is in $\mathrm{km} /$ mol. ss' and as' refer to modified ss (symmetric stretch) and as (antisymmetric stretch) modes: in $\mathrm{ss}^{\prime} \mathrm{s}\left(\mathrm{O}_{2} \mathrm{H}_{1}\right) / \mathrm{s}\left(\mathrm{O}_{2} \mathrm{H}_{3}\right) \approx 0.4 / 1.0$; in as' the ratio is reversed. The dimer has $C_{s}$ symmetry. ${ }^{b}$ Mode is mixed but contains mainly the $\mathrm{H}_{4}-\mathrm{O}_{5}-\mathrm{H}_{6}$ angle. ${ }^{c}$ Mode is mixed but contains mainly the $\mathrm{H}_{1}-\mathrm{O}_{2}-\mathrm{H}_{3}$ angle. ${ }^{d}$ Mode description differs from ab initio, m-SDFF being essentially the same as in water monomer. ${ }^{e}$ Mode description differs from ab initio (see text). 


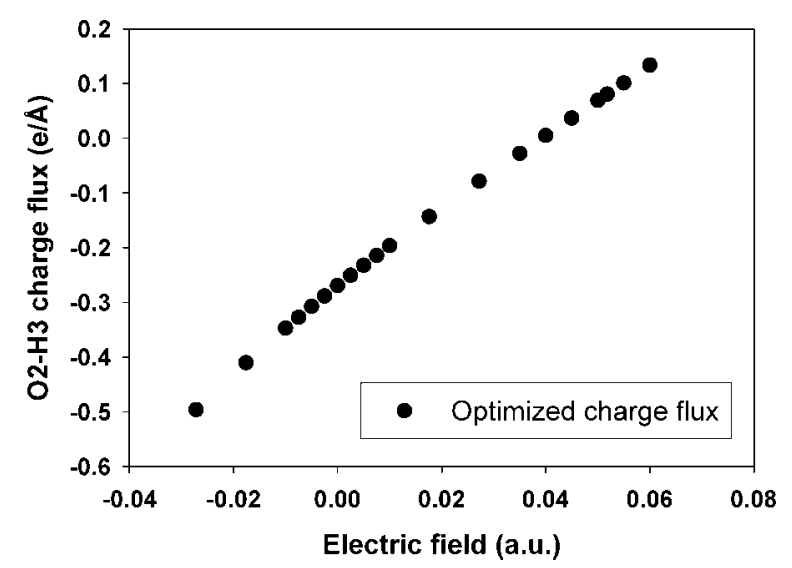

Figure 4. Optimized $\mathrm{O}_{2}-\mathrm{H}_{3}$ charge flux (in $e \AA^{-1}$ ) of fixed MP2/6$31++\mathrm{G}(\mathrm{d}, \mathrm{p})$ water monomer in homogeneous electric field (in atomic units) along the bond. Positive corresponds to the $\mathrm{O}_{2}-\mathrm{H}_{3}$ direction.

\subsubsection{Homogeneous Electric Field. Although the electric} field experienced by the donor molecule from the acceptor is not homogeneous, it is useful to consider first the uniform case since it can reveal major impacts of this interaction. Earlier studies of the water monomer in external homogeneous electric fields ${ }^{36,37}$ were focused on $\mathrm{OH}$ stretch frequency shifts and intensity changes induced by this interaction. We are additionally concerned with the physical impacts that such a field has on terms in the MM energy function, in particular on our model of the water monomer. To elucidate this, we studied two situations: the effect on a molecule of fixed structure, which reflects only the results of polarization by the electric field, and then the effect on a flexible one, which includes polarization as well as the results of structural changes due to the field.

In the first case, the water monomer was kept fixed at its MP2 equilibrium structure and placed in a homogeneous electric field parallel as well as antiparallel to the $\mathrm{O}_{2}-\mathrm{H}_{3}$ bond (perpendicular components have relatively little effect on the electrical properties of the bond ${ }^{37}$ ). This arrangement is also close to the substantive situation in the equilibrium water dimer, in which the hydrogen bond angle $\mathrm{O}-\mathrm{H} \cdots \mathrm{O}$ is close to $180^{\circ}$ $\left(174.8^{\circ}\right.$ at the MP2/6-31++G(d,p) level) and the field is directed close to the $\mathrm{O}_{2}-\mathrm{H}_{3}$ bond direction (about $10^{\circ}$ from this direction).

In considering how the induced electrical forces might be accounted for in an MM energy function, we evaluate the changes in the dipole derivatives as a function of the applied electric field. The molecular dipole moment is $\boldsymbol{\mu}=\boldsymbol{\mu}_{\text {stat }}+\boldsymbol{\mu}_{\text {ind }}$, where $\boldsymbol{\mu}_{\text {stat }}$ is the static dipole moment and $\boldsymbol{\mu}_{\text {ind }}=\alpha \mathbf{E}$ is the moment induced by the field, $\mathbf{E}$, as given by the molecular polarizability $\boldsymbol{\alpha}$. The dipole derivatives in Cartesian coordinates, $x_{k}$, are then

$$
\frac{\partial \mu}{\partial x_{k}}=\frac{\partial \mu_{\text {stat }}}{\partial x_{k}}+\frac{\partial \alpha}{\partial x_{k}} \mathbf{E}+\alpha \frac{\partial \mathbf{E}}{\partial x_{k}}
$$

We have examined two situations: in the first, we assume that $\partial \boldsymbol{\mu} / \partial x_{k} \equiv \partial \boldsymbol{\mu}_{\text {stat }} / \partial x_{k}$ and optimize charge fluxes to the ab initio dipole derivatives separately at each electric field. This is equivalent to implicitly absorbing the induction effect into the charge fluxes, i.e., having the charge fluxes emulate the static as well as the induced effects. (Of course, in the homogeneous field the $\alpha \partial \mathbf{E} / \partial x_{k}$ term is zero.) For this case, Figure 4 shows the $\mathrm{O}_{2}-\mathrm{H}_{3}$ bond charge flux as a function of the electric field. The following can be deduced: first, there is a slight asymmetry resulting from the noninclusion of a small hyperpolarizability;33

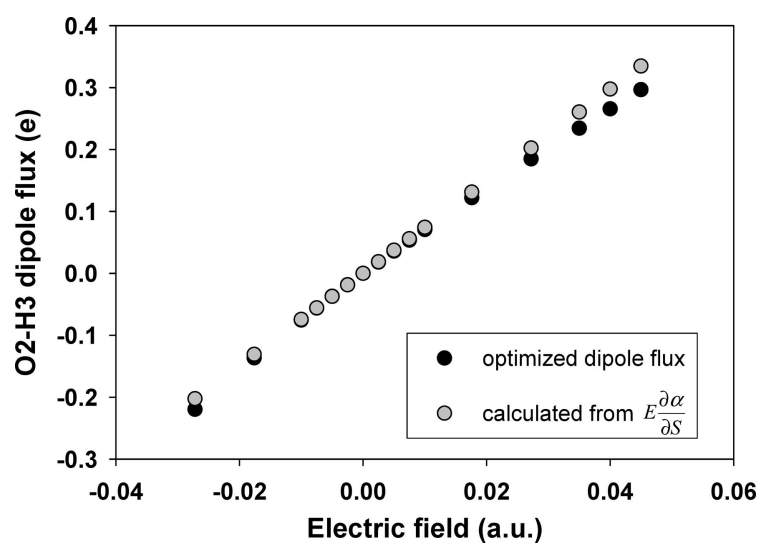

Figure 5. Comparison of MP2/6-31++G(d,p) fixed-monomer $\mathrm{O}_{2}-\mathrm{H}_{3}$ dipole flux (in $e$, black circles) and ab initio polarizability derivative term (gray circles). Positive corresponds to the $\mathrm{O}_{2}-\mathrm{H}_{3}$ direction.

second, the dependence of the charge flux on electric field is seen to be essentially linear for fields up to $\sim 0.01$ au $(0.51 \mathrm{~V} / \AA)$ and to depart from linearity for stronger fields; and third, the charge flux in parallel fields becomes less negative at stronger fields, eventually becoming positive at a field strength of $\sim 0.04$ au. We note that, utilizing our dimer model parameters (Table 1), the electric field strength at and along the $\mathrm{O}_{2}-\mathrm{H}_{3}$ bond is $0.0209 \mathrm{au}$, well within the range studied in Figure 4. The differences between the QM and SDFF dipole derivatives were found to be essentially zero up to the field strength of about $0.01 \mathrm{au}$, whereas without charge flux optimization, namely using only the monomer zero-field charge flux at each point, the deviations increase substantially with increasing electric field. Thus, the charge flux optimization yields good results, but the changes in parameters are unphysical and would be cumbersome to implement in an MM energy function.

A more physical picture would assume that the induced effects of the homogeneous electric field are explicitly accounted for by the $\mathbf{E} \partial \boldsymbol{\alpha} / \partial x_{k}$ terms. To test this, the charge fluxes were constrained to the isolated monomer values, and internal coordinate dipole fluxes ${ }^{10}$ were optimized to represent the induced part of the MP2 dipole derivatives at each electric field. Internal coordinate dipole fluxes, $\mathbf{d}_{j}$, describe the rate of change in the molecular dipole moment, $\boldsymbol{\mu}$, with deformation of internal coordinates, $S_{j}$, i.e.,

$$
\mathbf{d}_{j}=\frac{\partial \mu}{\partial S_{j}}
$$

so that

$$
\mu=\mu_{0}+\sum_{j} \mathbf{d}_{j}\left(S_{j}-S_{j 0}\right)
$$

where $\boldsymbol{\mu}_{0}$ is the dipole moment at zero deformation of the $S_{j}$. The dipole fluxes were introduced in the SDFF to account for vibrational IR intensities ${ }^{10}$ and they provide a very close connection to the quantities in eq 7 , but are not presently incorporated in the energy function. With the $\mathrm{OH}$ bond as $S_{j}$, we find from MP2 calculations on the isolated monomer that the component of $\partial \boldsymbol{\alpha} / \partial S_{j}$ along the bond is the most dominant element of that tensor, and has the value 7.44 au (Table 1). By calculating $\mathbf{d}_{j}=\mathbf{E} \partial \boldsymbol{\alpha} / \partial S_{j}$, and comparing them with the SDFF dipole flux values optimized along the $\mathrm{O}_{2}-\mathrm{H}_{3}$ bond to the dipole derivatives at the different fields, we can evaluate the accuracy of an energy function featuring the $\mathbf{E} \partial \boldsymbol{\alpha} / \partial S_{j}$ term. Figure 5 shows this comparison. The agreement is almost exact up to a $\sim 0.01$ 
au field strength, the optimized dipole flux parameter showing a slight nonlinearity at higher field strengths.

We conclude that the $\mathbf{E} \partial \boldsymbol{\alpha} / \partial S_{j}$ term basically accounts for the dipole derivative behavior in the homogeneous electric field, but that, since the present SDFF does not provide for dipole fluxes or polarizability derivatives in the energy function, the optimized charge fluxes can provide a suitable emulation of the induced effects. In this sense, they may be considered effective charge fluxes, although their detailed physical origin still needs to be established.

Before examining the results in an inhomogeneous field, we tested the effect of geometric flexibility on the electric field behavior of the charge flux by optimizing the structure of the water monomer as a function of the homogeneous field. In this case the field is automatically along the dipole moment direction, i.e., the bisector. The optimized $\mathrm{O}-\mathrm{H}$ charge fluxes derived from the $a b$ initio dipole derivatives of the energy-minimized molecule differ relatively little from those of the molecule at the fixed geometry (for example, at a field strength of 0.0272 au the $\mathrm{O}_{2}-\mathrm{H}_{3}$ charge flux for the minimized molecule is $-0.1512 e \AA^{-1}$ whereas the comparable value for the fixed molecule is $-0.1473 e \AA^{-1}$ ) and the molecular property agreement with ab initio is very good.

3.2.2.2. Inhomogeneous Electric Field. In the actual water dimer, the donor molecule of course does not reside in a homogeneous but in an inhomogeneous electric field. It is therefore of interest to determine the relative effects of the field, $\mathbf{E}$, and the field gradient, $\partial \mathbf{E} / \partial x_{k}$ (see eq 7 ), on the properties of the donor, particularly on the effective charge flux of the hydrogen-bonded $\mathrm{O}_{2}-\mathrm{H}_{3}$ bond. We examined this by placing a donor molecule in the LP/ILP monomer charge distribution of a dimer-positioned acceptor molecule (a so-called external charge distribution, ECD). Although the acceptor charge distribution would differ somewhat due to the polarization by the donor, this effect is thought to be relatively small. ${ }^{38}$ This is also supported by our finding that the natural population analysis (NPA) charges on the acceptor change by only $\sim 1 \%$ to $3 \%$ from those of the monomer. In these (QM and SDFF) calculations the system was constrained to the MP2 energy-minimized structure of the dimer, with only the $\mathrm{H}_{3} \cdots \mathrm{O}_{5}$ distance being varied. For maximum agreement with the corresponding QM calculations (i.e., monomer in a fixed point charge distribution), the LP/ILP electrical model without size but with group polarization was used for the donor molecule while the same monomer LP/ILP charges as in the QM calculation were used for the acceptor molecule.

Since in the SDFF the monomer in the ECD has to be treated similarly to the dimer and because the QM calculations give only monomer (in this case donor) properties, the dipole derivatives for the acceptor were calculated using our program and the above-explained LP/ILP charge distributions of the donor and acceptor. The optimization of the charge flux parameters of the monomer-ECD system to this combined MP2LP/ILP dipole derivative matrix yields a diagonal $\mathrm{O}_{2}-\mathrm{H}_{3}$ charge flux parameter of $-0.1227 e \AA^{-1}$, compared to the value of $-0.2696 e \AA^{-1}$ for that optimized to the water monomer. The acceptor LP/ILP average electric field along the donor $\mathrm{O}_{2}-\mathrm{H}_{3}$ bond is $0.0209 \mathrm{au}$, and, assuming that this is representative of the effect of the field alone, by using the charge flux values from Figure 4 for the homogeneous field component, -0.1209 $e \AA^{-1}$, the field gradient contribution to the change in charge flux is therefore -0.0018 e $\AA^{-1}(-0.1227-[-0.1209])$. Compared to the homogeneous field contribution, viz., +0.1487 $e \AA^{-1}(-0.1209-[-0.2696])$, the gradient contribution is seen

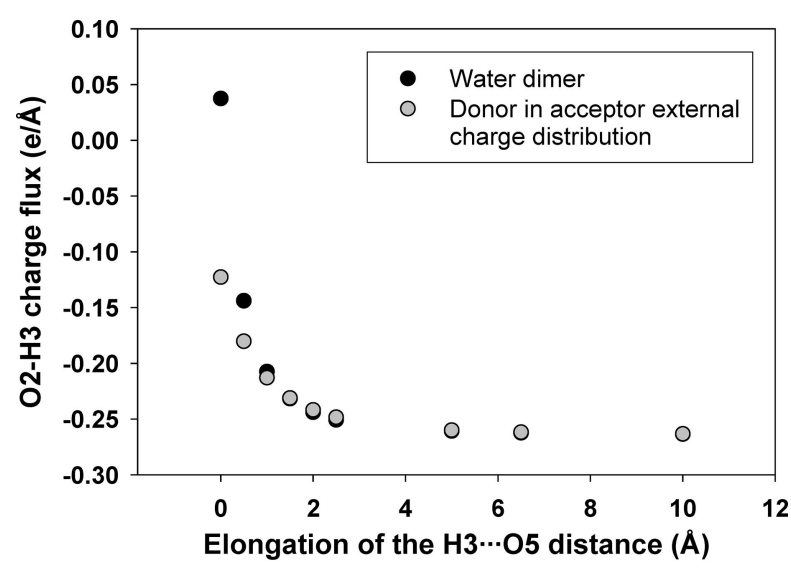

Figure 6. Optimized $\mathrm{O}_{2}-\mathrm{H}_{3}$ charge fluxes (in $e \AA^{-1}$ ) as a function of the elongation from the MP2/6-31++ $\mathrm{G}(\mathrm{d}, \mathrm{p})$ minimized $\mathrm{H}_{3} \cdots \mathrm{O}_{5}$ distance (in $\AA$ ) for the SDP-LP/ILP dimer (black circles) and the polarizable LP/ILP electrical model without size donor in the nonpolarizable LP/ILP without size external charge distribution of the acceptor (gray circles). Excluding the $\mathrm{H}_{3} \cdots \mathrm{O}_{5}$ distance, the structures were constrained to the MP2/6-31++G(d,p) optimized dimer structure.

to be quantitatively small. (If, because of its closeness to the polarization center, ${ }^{33}$ we use the field at $\mathrm{O}_{2}, 0.0093 \mathrm{au}$, the bond charge flux is $-0.2015 e \AA^{-1}$ and the field gradient contribution is $+0.0788 e \AA^{-1}$ compared to a field contribution of +0.0681 $e \AA^{-1}$.) Aside from the issue of such relative values, it is noteworthy that the combined electric field effects have increased the charge flux by an effective nontrivial $0.1469 e$ $\AA^{-1}(-0.1227-[-0.2696])$.

3.2.2.3. Wave Function Overlap. Despite including homogeneous and inhomogeneous field components in the monomerECD system, we still find that the calculated intensity of the $v_{9}$ stretch mode, $80.1 \mathrm{~km} / \mathrm{mol}$, is in significant disagreement with the ab initio dimer value, $261.7 \mathrm{~km} / \mathrm{mol}$, even though the intensity of the other stretch mode, $v_{11}, 134.8 \mathrm{~km} / \mathrm{mol}$, is in reasonable agreement with the ab initio value, $120.9 \mathrm{~km} / \mathrm{mol}$ (Table 3). As previously noted, the full water dimer $\mathrm{O}_{2}-\mathrm{H}_{3}$ charge flux optimized to the MP2 dipole derivatives is +0.0339 $e \AA^{-1}$ (Table 1), an effective increase of $+0.1566 e \AA^{-1}$ over the ECD value $\left(-0.1227 e \AA^{-1}\right)$. We also find that the intensity of $v_{9}$ has now increased to $223.3 \mathrm{~km} / \mathrm{mol}$, in reasonable agreement with the ab initio value, $261.7 \mathrm{~km} / \mathrm{mol}$, while the intensity of $v_{11}$ is not significantly affected over the ECD value (Table 3). This increase in the $\mathrm{O}_{2}-\mathrm{H}_{3}$ charge flux is clearly a result of the overlap of acceptor and donor wave functions in the formation of the QM hydrogen bond, and although we refer to it as an overlap charge flux (OCF) it does not necessarily describe any real flow of charge but only emulates a dipole flux caused by a deformation of the charge distribution in dimer formation. The OCF reduces to practically zero, leaving just the electrical contribution, by $\sim 1$ to $1.5 \AA$ separation of the monomers from equilibrium (an observation also noted by others ${ }^{39}$ ), as is shown in Figure 6 for the dimer as well as the ECD case as a function of the $\mathrm{H}_{3} \cdots \mathrm{O}_{5}$ separation.

Dipole fluxes (or charge fluxes that emulate them) optimized to the dimer dipole derivatives cannot directly be assigned locations in the system. However, the physical origin of the OCF can be further elucidated by evaluating changes in the electric-potential derived charges as a function of bond deformation. In Figure 7 we show the CHELPG charges on the $\mathrm{H}_{3}$ atom, as given by Gaussian 03 in a fixed monomer and a fixed dimer, as a function of elongation of the $\mathrm{O}_{2}-\mathrm{H}_{3}$ bond (i.e., $\mathrm{H}_{3}$ being the only atom moving). The initial slopes of these curves 


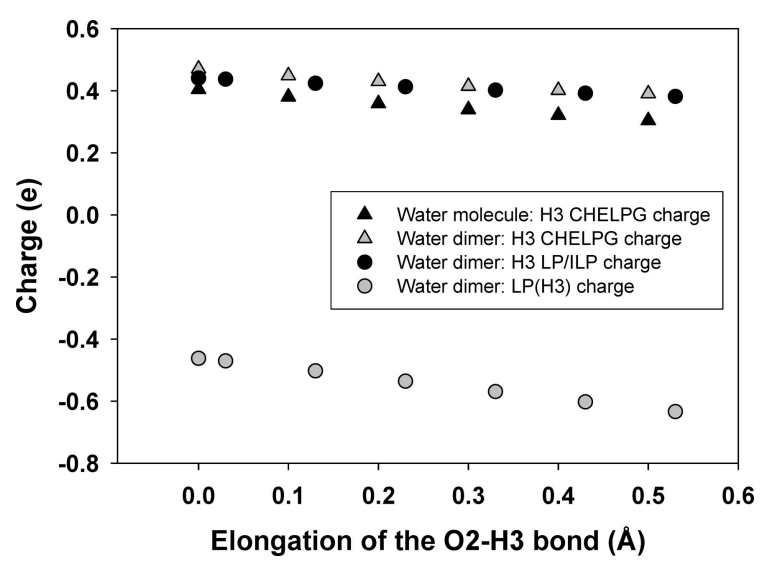

Figure 7. Charges (in $e$ ) as a function of elongation from the MP2/ 6-31 $++\mathrm{G}(\mathrm{d}, \mathrm{p})$ optimized $\mathrm{O}_{2}-\mathrm{H}_{3}$ bond length (in $\AA$ ). Upper symbols: $\mathrm{H}_{3}$ MP2/6-31++G(d,p) CHELPG charges of the monomer (black triangles); $\mathrm{H}_{3} \mathrm{MP} 2 / 6-31++\mathrm{G}(\mathrm{d}, \mathrm{p}) \mathrm{CHELPG}$ charges of the dimer (gray triangles); $\mathrm{H}_{3}$ SDP-LP/ILP charges (see text) by setting all $\mathrm{O}_{2}-\mathrm{H}_{3}$ bond charge fluxes to zero (black circles). Lower symbols (gray circles): Corresponding SDP-LP/ILP charges of the off-atom LP site facing $\mathrm{H}_{3}$, $\mathrm{LP}\left(\mathrm{H}_{3}\right)$. Excluding the $\mathrm{O}_{2}-\mathrm{H}_{3}$ bond, the molecules have been fixed to their MP2/6-31++G(d,p) structures.

represent the diagonal $\mathrm{O}_{2}-\mathrm{H}_{3}$ CHELPG charge fluxes in an unrestricted atomic point charge model: for the monomer this is $-0.2163 e \AA^{-1}$ and for the dimer it is $-0.2020 e \AA^{-1}$ (compared to our optimized SDFF monomer value of -0.2696 $\left.e \AA^{-1}\right)$. The near equality of the dimer CHELPG charge flux to that of the monomer shows that the intrinsic monomer parameters are transferable to the dimer, and the fact that complete polarization, represented by an incremental ECD-induced effective charge flux, viz., $+0.1469 e \AA^{-1}$, does not fully account for the final required $+0.0339 e \AA^{-1}$ effective $\mathrm{O}_{2}-\mathrm{H}_{3}$ charge flux in the dimer indicates that the OCF must be associated with hydrogen bond-induced changes resulting from the QM interactions between donor and acceptor. The underlying question is the physical origin of this OCF, and for the answer it is necessary to inquire into the changes in the acceptor partner in the hydrogen bond. We do this by directly observing the changes in the atomic and off-atom site charges in a fit to the dimer electric potential as a function of the $\mathrm{H}_{3} \cdots \mathrm{O}_{5}$ distance $\left(\mathrm{H}_{3}\right.$ again being the only atom moving). Thus, using our own method $^{17}$ and the geodesic point selection scheme for the calculation of the electric potential, ${ }^{40}$ we have calculated the charges with $\mathrm{O}_{2}-\mathrm{H}_{3}$ bond elongation in the MP2-minimized dimer by utilizing our monomer electrical model but setting all $\mathrm{O}_{2}-\mathrm{H}_{3}$ charge fluxes to zero. The charges on $\mathrm{H}_{3}$ and on the $\mathrm{O}_{5}$ LP site facing $\mathrm{H}_{3}$ (named $\mathrm{LP}\left(\mathrm{H}_{3}\right)$ ) are shown in Figure 7. (Other charge fluxes, except those related to the $\mathrm{O}_{2}-\mathrm{H}_{3}$ bond, are very similar in monomer and dimer.) The changes in the charge on $\mathrm{LP}\left(\mathrm{H}_{3}\right)$ demonstrate that hydrogen bonding also leads to reorganization of charge on the acceptor. If we take the initial slope of this change in charge as an $\mathrm{LP}\left(\mathrm{H}_{3}\right)-\mathrm{O}_{5}$ charge flux due to elongation of the $\mathrm{O}_{2}-\mathrm{H}_{3}$ bond, viz., $-0.313 e \AA^{-1}$, and transform it into a dipole flux (which is closer to a basic QM quantity), viz., $\left|\mathbf{d}_{\mathbf{L P}}\right|=\mathrm{R}_{\mathrm{LP}} a_{\mathrm{LP}}=0.43(0.313)=0.135 e$, then we find that this is in rough agreement with the OCF transformed to a dipole flux, viz., $\left|\mathbf{d}_{\text {OCF }}\right|=R_{\mathrm{O}_{2 \mathrm{H}} 3} a_{\mathrm{OCF}}=$ $0.97(0.157)=0.152 \mathrm{e}$. The notion of a dipole flux on the acceptor is also supported by electron density difference plots, which show significant changes around $\mathrm{O}_{5},{ }^{41,42}$ with the atomic dipole on $\mathrm{O}_{5}$ found to change from 0.1309 au in the monomer to 0.1939 au in the dimer. ${ }^{39}$

Although it has been proposed that the significant change in the mainly $\mathrm{O}_{2}-\mathrm{H}_{3}$ stretch IR intensity arises from dynamic effects that can be attributed to a so-called charge transfer charge flux, ${ }^{38}$ the results described above lead us to believe that most of the OCF can be explicitly attributed to an intermolecular dipole flux on the acceptor. Such a flux is not surprising since hydrogen bonding is expected to lead to changes in the properties of both donor and acceptor. The OCF contribution is expected to be comparable to that from polarization, ${ }^{38}$ which we also find for our MP2/6-31++G(d,p) SDFF model: the change from the ECD alone, $0.1469 e \AA^{-1}$, is similar to that from the $\mathrm{OCF}, 0.1566 e \AA^{-1}$.

The challenge will be to incorporate such intermolecular dipole flux changes on hydrogen bonding into an MM force field in a physically meaningful way. It is clear that a new kind of addition to the classical MM function is needed if the donor and acceptor electrical properties are to be accurately described. In particular, it will be necessary to account for the variation of this acceptor flux with $\mathrm{H} \cdot \cdots \mathrm{O}$ distance as well as angle on the basis of the QM-determined relationship. This should be feasible, and can be expected to give a much more physically reliable description of the properties of the moieties that participate in a hydrogen bond.

\section{Ab Initio Agreement of the SDP-LP/ILP Water Model}

4.1. Monomer SDP-LP/ILP Water. The parameters resulting from the incorporation of the components of our SDFF treatment of the water monomer are given in Table 1, with the minimized monomer properties of our SDP-LP/ILP model being presented in Table 2. As previously noted, the model gives very good monomer electrical properties: the electric potential around the monomer has an rrms deviation of $0.95 \%$, the dipole moment agreement is excellent (the ab initio moment is somewhat high for our basis set in comparison with values ranging from $2.04 \mathrm{D}^{43}$ to $1.98 \mathrm{D}^{44}$ for more extensive basis sets), and the quadrupole moments (the latter are given in the traceless and nonzero-trace matrix forms to emphasize the necessary nonzero $Q_{z z}$ elements) are very good. Since the valence parameters and charge fluxes are derived directly from analytically transformed QM results, the monomer frequencies and IR intensities agree essentially exactly with the ab initio results. We note that, because there is no intramolecular nonbond potential for isolated monomer water, the SDFF force constants, and therefore the frequencies, are independent of the electric model. ${ }^{1}$ However, the IR intensities are not model independent, and therefore the charge fluxes for different electrical models can be different (see Table S1 in the Supporting Information).

A further assessment of the performance of this model can be gained from the results in which a water monomer was energy minimized in varying electric fields along the bisector. First, all charge flux parameters were constrained to the monomer value, then they were optimized at each electric field to the MP2 dipole derivatives to include induced effects, and in both cases various monomer properties were examined as a function of the field. A comparison of the MP2 and SDFF results is given in Table 4 for the zero and the largest field used, viz., $0.0272 \mathrm{au}$. If charge fluxes are constrained to the monomer value at each electric field (SDFF2), the agreement for structural parameters and frequencies is comparable to the case of individually optimized charge fluxes (SDFF1), but the intensity agreement is (and therefore the force agreement must $\mathrm{be}^{2}$ ) significantly poorer (essentially maintaining values with field that are equal to the zero-field case). As discussed earlier, the monomer SDFF2 (i.e., SDP-LP/ ILP) model augmented by the polarizability derivative terms 
TABLE 4: Variation of Water Monomer Properties ${ }^{a}$ with Electric Field ${ }^{b}$

\begin{tabular}{llllllll}
\hline & \multicolumn{3}{c}{$E=0$} & & \multicolumn{3}{c}{$E=0.0272$} \\
\cline { 2 - 5 } \cline { 6 - 8 } & MP2 & SDFF1 & SDFF2 & & MP2 & SDFF1 & SDFF2 \\
\hline$R$ & 0.9634 & 0.9634 & 0.9634 & & 0.9685 & 0.9698 & 0.9669 \\
$\theta$ & 105.35 & 105.35 & 105.35 & & 101.57 & 101.27 & 101.93 \\
$\mu$ & 2.2279 & 2.2277 & 2.2277 & & 2.6710 & 2.7641 & 2.7361 \\
$v(\mathrm{HOH})$ & 1619.3 & 1619.5 & 1619.5 & & 1695.0 & 1706.3 & 1717.1 \\
$I(\mathrm{HOH})$ & 88.7 & 88.7 & 88.7 & & 109.0 & 107.0 & 74.3 \\
$v(\mathrm{OH}$ ss $)$ & 3863.5 & 3863.3 & 3863.3 & & 3823.7 & 3793.1 & 3841.1 \\
$I(\mathrm{OH} \mathrm{ss})$ & 8.6 & 8.6 & 8.6 & & 46.8 & 47.5 & 8.1 \\
$\nu(\mathrm{OH}$ as $)$ & 4010.3 & 4010.1 & 4010.1 & & 3923.3 & 3923.1 & 3969.2 \\
$I(\mathrm{OH}$ as $)$ & 63.3 & 63.3 & 63.3 & & 121.5 & 120.4 & 63.1
\end{tabular}

${ }^{a} R=\mathrm{OH}$ bond length in $\AA$; $\theta=\mathrm{HOH}$ angle in deg; $\mu=$ dipole moment in debye; $v=$ frequency in $\mathrm{cm}^{-1}$; ss $=$ symmetric stretch, as $=$ antisymmetric stretch; $I=\mathrm{IR}$ intensity in $\mathrm{km} / \mathrm{mol} .{ }^{b} E=$ electric field in au; MP2 = MP2/6-31++G(d,p); SDFF1 = SDP-LP/ILP model with charge fluxes at each $E$ optimized to MP2 dipole derivatives; SDFF2 $=$ SDP-LP/ILP model with charge fluxes at each $E$ kept at $E=0$ value.

$\left(\mathbf{E} \partial \boldsymbol{\alpha} / \partial S_{j}\right)$ in the energy function would result in performance effectively equal to SDFF1. It is worth noting that the frequencies at the nonzero fields have been calculated with the SDFF monomer intrinsic force constants. In principle this can account for the frequency disagreement even for SDFF1, since just as forces in an electric field derive from the first derivative of the potential energy, and thus depend on the field and its gradient, ${ }^{2}$ the force constants derive from the second derivative and will be influenced by the field, its gradient, and its second derivative. We have found that exact agreement with the MP2 frequencies can be obtained with relatively minor changes in force constants, but since these were determined manually and not from knowledge of the explicit field dependences we have chosen not to implement this change.

4.2. Dimer SDP-LP/ILP Water. In Table 3 we present the properties of the SDP-LP/ILP dimer, both with monomer charge fluxes (m-SDFF) and with dimer charge fluxes (dSDFF), the latter that, because the actual dipole flux components are not currently implemented in our SDFF energy function, have had charge fluxes individually optimized to dimer ab initio dipole derivatives in order to implicitly include polarization and QM wave function overlap effects (the OCF). The properties of the m-SDFF dimer, except for the order of $v_{9}$ and $v_{10}$ as well as of $v_{11}$ and $v_{12}$ and the value of $I_{9}$, are substantively the same as those of the d-SDFF dimer (which makes this a strong water model in its own right). As noted before, although most of the d-SDFF dimer charge fluxes have not changed dramatically from those of the isolated monomer, the diagonal $\mathrm{O}_{2}-\mathrm{H}_{3}$ flux has undergone a change, from a value of $-0.2696 e \AA^{-1}$ to an effective value of $+0.0339 e \AA^{-1}$. A comparison of its detailed structural properties with the corresponding ab initio results is quite good. The dipole moment is well reproduced (although again the ab initio value is high, $3.22 \mathrm{D}$, compared to a result with a higher basis set, $2.76 \mathrm{D}^{43}$ ). Some features can bear improvement (e.g., the $\mathrm{H}_{3} \cdots \mathrm{O}_{5}$ distance and the $\mathrm{HOH}$ angles). The frequencies are generally well reproduced, with the most problematic being $v_{6}$ (however, higher level $\mathrm{ab}$ initio computations give a lower value, e.g., $640 \mathrm{~cm}^{-1} 45$ and even $600 \mathrm{~cm}^{-1},{ }^{46}$ as well as when BSSEs are taken into account), and the IR intensities (considering their greater degree of sensitivity) are respectably predicted (except for the hydrogen bond stretch, $v_{4}$, whose present SDFF mode

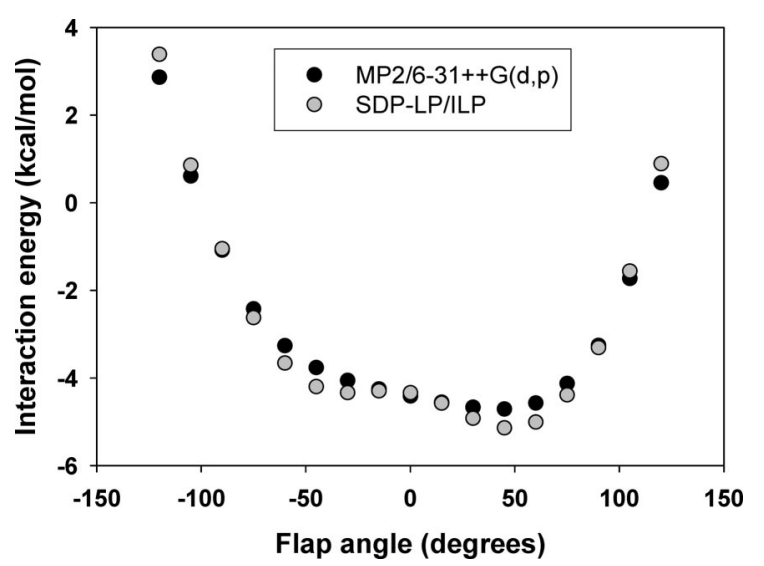

Figure 8. Interaction energy of MP2/6-31++G(d,p) fixed-monomer dimer (in $\mathrm{kcal} / \mathrm{mol}$ ) as a function of flap angle, $\mathrm{H}_{3} \cdots \mathrm{H}_{4}-\mathrm{H}_{6}-\mathrm{O}_{5}$ (in $\mathrm{deg}$ ), at the MP2/6-31++G(d,p) level (black circles) and with our SDPLP/ILP model (gray circles).

description is significantly more $\mathrm{H}_{3} \cdots \mathrm{O}_{5}$ stretch intensive than the QM mode, which our tests have ascertained to be related to the $\mathrm{O}_{5} \mathrm{vdW}$ shift). We expect that fine-tuning of the model (i.e., SDP-LP/ILP including polarizability derivatives and the intermolecular $\mathrm{O}-\mathrm{H} \cdots \mathrm{LP}-\mathrm{O}$ dipole flux), probably based on higher level ab initio calculations, should improve these relatively small discrepancies.

We have examined two other features of our SDP-LP/ILP water model that bear on its predictive capabilities. In the first case, we examined the energy difference between the coplanar and perpendicular fixed-monomer $\mathrm{H}_{2} \mathrm{O} \cdots \mathrm{OH}_{2}$ configurations as a function of the $\mathrm{O} \cdots \mathrm{O}$ distance, which should be a test of the electrical anisotropy of the model. The comparisons with the ab initio results were shown in Figure 2. The agreement at large separations is not surprising, but the relatively good agreement at short distances is gratifying. Of course, the agreement could be made perfect if only this test were applied, for example by changing slightly the oxygen off-nucleus vdW location; the present position, however, was optimized simultaneously to these as well as many other dimer configurations, and thus represents an overall test. (A similar comment is relevant to the discrepancy in $E_{\text {int }}$ : without fitting simultaneously, as we have done, to many different configurations, better agreement near the minimum could be achieved, although with the result of worse (but less visible) agreement elsewhere. We have avoided this, in the belief that future work can improve on this property of the model without compromising on the inclusion of the OCF.) In the second case, we calculated the variation in the interaction energy of the fixed-monomer dimer as a function of the flap angle, viz., the out-of-plane angle $\mathrm{H}_{3} \cdots \mathrm{H}_{4}-\mathrm{H}_{6}-\mathrm{O}_{5}{ }^{20}$ The comparisons with the ab initio results are shown in Figure 8, and agreement is seen to be excellent over the entire range, and much better than those of the TIP4P and TIP5P water models that have been developed for the condensed phase. ${ }^{20}$

\section{Conclusions}

As we have emphasized, ${ }^{2}$ accurate prediction of forces, beyond just structure and energy agreement, is needed to provide physical reliability in structure and dynamics predictions, which in turn requires incorporation of accurate charge and polarizability fluxes in MM energy functions. To achieve such electrical completeness and as critical tests of the energy function, vibrational properties such as modes, frequencies, and intensities must be satisfactorily reproduced, i.e., the force field must be 
"spectroscopically determined". Implementation of this approach ${ }^{1}$ has now led us to the development of an SDFF for the water monomer and dimer and, in the process, to evidence for the greater transferability of parameters and for the need to incorporate a treatment of the hydrogen bond interaction in force fields that goes beyond the canonical MM protocol.

We find that the requirements for monomer SDFF water, in the context of producing agreement with QM structural, electrical (dipole, quadrupole, and potential), and vibrational (mode, frequency, and intensity) properties, must include a number of features in the model: an off-plane charge distribution, this distribution consisting of four off-atom charge sites, two in traditional lone pair (LP) and two in inverted lone pair (ILP) positions; allowance for a diffuse size to these off-atom charges; and the SDFF incorporation of charge fluxes (i.e., the change in charge with change in internal coordinate). Such an electrical model (Table 1), whose parameters are determined by optimization to the ab initio electric potential and dipole derivatives, together with the SDFF-transformed valence force field, ${ }^{1}$ gives an almost exact reproduction of monomer ab initio (in this case MP2/6-31++G(d,p)) properties. Including polarization and the van der Waals parameters (Table 1), we call this a Spectroscopically Determined Polarizable LP/ILP, SDPLP/ILP, model.

The incorporation of the monomer electrical LP/ILP model into the SDFF of the dimer involves the addition of three elements. The first two are standard: the van der Waals potential, which we find most suitable in the L-J 9-6 form, and whose parameters are determined from BSSE-corrected interaction energies of multiple configurations of fixed-monomer dimers and include an offset of the oxygen vdW center; and polarization, which we include in the form of a recently developed group polarizability model ${ }^{33}$ together with polarizability derivatives. ${ }^{2}$ The third element is the special treatment needed to describe the effects of the hydrogen bond interaction, viz., the overlap of the donor and acceptor wave functions.

The evidence for the latter required departure from the canonical form of the MM function is the fact that simply carrying over the monomer parameters together with polarization and $\mathrm{vdW}$ interactions does not satisfactorily reproduce all of the SDFF-important QM results, outstandingly the huge IR intensity increase in a donor hydrogen-bonded $\mathrm{OH}$ stretch mode. This intensity is primarily determined by the effective charge flux in the hydrogen-bonded $\mathrm{OH}$ bond, which has a value of $-0.2696 e \AA^{-1}$ in the monomer, increases by $0.1469 e \AA^{-1}$ to a value of $-0.1227 e \AA^{-1}$ as a result of the classical induced effects from the electric field of the LP/ILP acceptor external charge distribution (ECD), but still does not attain the needed effective value of +0.0339 e $\AA^{-1}$ in the actual dimer required to yield the ab initio predicted IR intensity. However, we show that in our electrical model the intrinsic monomer bond charge flux does not change in the dimer, thus demonstrating that MM monomer properties are indeed transferable to the dimer. The additional $+0.1566 e \AA^{-1}$ effective charge flux that must be incorporated upon hydrogen bonding actually originates physically from an intermolecular dipole flux on the acceptor. (Although such terms are not currently implemented in our energy function, we have shown that they can be emulated by an "effective" charge flux on the donor hydrogen-bonded $\mathrm{OH}$ bond.) We designate this additional flux over the induced effects as an overlap charge flux, OCF, since it arises as a result of the overlap of wave functions in the formation of the hydrogen bond. Its value, as suggested, ${ }^{38}$ is approximately equal to that of the ECD polarization contribution.
Such an OCF term is not at present included in the standard formalism of an MM function, but it can be expected to be incorporated relatively routinely. It is obviously of basic importance in describing the changed electrical properties of the donor and acceptor in the dimer and its inclusion is an important first step in developing a more physical description of condensed-phase water (obviously necessarily based on further studies of multiply hydrogen-bonded molecules). At present, it gives our SDFF water model the maximum currently available QM compatibility with maximum transferability of monomer parameters. This physical OCF component clearly could have fundamental impacts in the treatment of other hydrogen bond interactions, probably with great significance for that of the peptide group. ${ }^{47}$ The fact that such a distinctively QM-unique property can be modeled in MM energy functions gives encouragement that other basic ab initio features could be substantively represented in such force fields.

Acknowledgment. This research was supported by NSF Grant No. MCB0212232. Additional funding was provided by the Michigan Universities Commercialization Inititiative. The results of this research were presented at the 40th Midwest Theoretical Chemistry Conference, Ann Arbor, MI, June 26-28, 2008.

Supporting Information Available: Tables of parameters and properties of nonpolarizable SDFF water monomer (Table S1) and water dimer (Table S2) force fields for the following models: charge, charge/dipole, inverted lone pair, lone pair/ inverted lone pair, and lone pair/inverted lone pair with offatom size. This material is available free of charge via the Internet at http://pubs.acs.org.

\section{References and Notes}

(1) Palmo, K.; Mannfors, B.; Mirkin, N. G.; Krimm, S. Biopolymers 2003, 68, 383-394.

(2) Palmo, K.; Mannfors, B.; Mirkin, N. G.; Krimm, S. Chem. Phys. Lett. 2006, 429, 628-632.

(3) Feynman, R. P. Phys. Rev. 1939, 56, 340-343.

(4) Palmo, K.; Pietila, L.-O.; Krimm, S. J. Comput. Chem. 1991, 12, 385-390.

(5) Palmo, K.; Mirkin, N. G.; Pietila, L.-O.; Krimm, S. Macromolecules 1993, 26, 6831-6840.

(6) Palmo, K.; Mirkin, N. G.; Krimm, S. J. Phys. Chem. A 1998, 102, 6448-6456.

(7) Mannfors, B.; Sundius, T.; Palmo, K.; Pietila, L.-O.; Krimm, S. J. Mol. Struct. 2000, 521, 49-75.

(8) Palmo, K.; Krimm, S. J. Polym. Sci.: Part B: Polym. Phys. 1996, 34, 37-45.

(9) Palmo, K.; Krimm, S. Macromolecules 2002, 35, 394-402.

(10) Palmo, K.; Krimm, S. J. Comput. Chem. 1998, 19, 754-768.

(11) Frish, M. J.; Trucks, G. W.; Schlegel, H. B.; Scuseria, G. E.; Robb, M. A.; Cheeseman, J. R.; Zakrzewski, V. G.; Montgomery, J. A., Jr.; Stratmann, R. E.; Burant, J. C.; Dapprich, S.; Millam, J. M.; Daniels, A. D.; Kudin, K. N.; Strain, M. C.; Farkas, O.; Tomasi, J.; Barone, V.; Cossi, M.; Cammi, R.; Mennucci, B.; Pomelli, C.; Adamo, C.; Clifford, S.; Ochterski, J.; Petersson, G. A.; Ayala, P. Y.; Cui, Q.; Morokuma, K.; Malick, D. K.; Rabuck, A. D.; Raghavachari, K.; Foresman, J. B.; Cioslowski, J.; Ortiz, J. V.; Stefanov, B. B.; Liu, G.; Liashenko, A.; Piskorz, P.; Komaromi, I.; Gomperts, R.; Martin, R. L.; Fox, D. J.; Keith, T.; Al-Laham, M. A.; Peng, C. Y.; Nanayakkara, A.; Gonzalez, C.; Challacombe, M.; Gill, P. M. W.; Johnson, B.; Chen, W.; Wong, M. W.; Andres, J. L.; Gonzalez, C.; Head-Gordon, M.; Replogle, E. S.; Pople, J. A. GAUSSIAN 98, Revision A.3; Gaussian, Inc.: Pittsburgh, PA, 1998.

(12) Frish, M. J.; Trucks, G. W.; Schlegel, H. B.; Scuseria, G. E.; Robb, M. A.; Cheeseman, J. R.; Montgomery, J. A., Jr.; Vreven, T.; Kudin, K. N.; Burant, J. C.; Millam, J. M.; Iyengar, S. S.; Tomasi, J.; Barone, V.; Mennucci, B.; Cossi, M.; Scalmani, G.; Rega, N.; Petersson, G. A.; Nakatsuji, H.; Hada, M.; Ehara, M.; Toyota, K.; Fukuda, R.; Hasegawa, J.; Ishida, M.; Nakajima, T.; Honda, Y.; Kitaot, O.; Nakai, H.; Klene, M.; Li, X.; Knox, J. E.; Hratchian, H. P.; Cross, J. B.; Adamo, C.; Jaramillo, J.; Gomperts, R.; Stratmann, R. E.; Yazyev, O.; Austin, A. J.; Cammi, R.; Pomelli, C.; Ochterski, J. W.; Ayala, P. Y.; Morokuma, K.; Voth, G. A.; Salvador, P.; Dannenberg, J. J.; Zakrzewski, V. G.; Dapprich, S.; Daniels, A. D.; Strain, M. C.; Farkas, O.; Malick, D. K.; 
Rabuck, A. D.; Raghavachari, K.; Foresman, J. B.; Ortiz, J. V.; Cui, Q.; Baboul, A. G.; Clifford, S.; Cioslowski, J.; Stefanov, B. B.; Liu, G.; Liashenko, A.; Piskorz, P.; Komaromi, I.; Martin, R. L.; Fox, D. J.; Keith, T.; Al-Laham, M. A.; Peng, C. Y.; Nanayakkara, A.; Challacombe, M.; Gill, P. M. W.; Johnson, B.; Chen, W.; Wong, M. W.; Gonzalez, C.; Pople, J. A. Gaussian 03, Revision C.02; Gaussian, Inc.: Wallingford, CT, 2004.

(13) Schmidt, M. W.; Baldridge, K. K.; Boatz, J. A.; Elbert, S. T.; Gordon, M. S.; Jensen, J. H.; Koseki, S.; Matsunaga, N.; Nguyen, K. A.; Su, S. J.; Windus, T. L.; Dupuis, M.; Montgomery, J. A. J. Comput. Chem. 1993, 14, 1347-1363.

(14) Boys, S. F.; Bernardi, F. Mol. Phys. 1970, 19, 553-566.

(15) Simon, S.; Duran, M.; Dannenberg, J. J. J. Chem. Phys. 1996, 105, 11024-11031.

(16) Bende, A.; Knapp-Mohammady, M.; Suhai, S. Int. J. Quantum Chem. 2003, 92, 152-159.

(17) Mannfors, B.; Palmo, K.; Krimm, S. J. Mol. Struct. 2000, 556 $1-21$

(18) Mannfors, B.; Mirkin, N. G.; Palmo, K.; Krimm, S. J. Comput. Chem. 2001, 22, 1933-1943.

(19) Williams, D. E. In Reviews in Computational Chemistry; Lipkowitz, K. B., Boyd, D. B., Eds.; VCH Publishers: New York, 1991; Vol. 2, pp 219-271.

(20) Mahoney, M. W.; Jorgensen, W. L. J. Chem. Phys. 2000, 112, 8910-8922.

(21) Sagui, C.; Pomorski, P.; Darden, T. A.; Roland, C. J. Chem. Phys. 2004, 120, 4530-4544.

(22) Pacios, L. F. In Hydrogen Bonding-New Insights; Grabowski, S. J., Ed.; Springer: New York, 2006; pp 109-148.

(23) Singh, U. C.; Kollman, P. A. J. Comput. Chem. 1984, 5, 129-145.

(24) Robert Krasny, personal communication

(25) Lindsay, K.; Krasny, R. J. Comput. Phys. 2001, 172, 879-907.

(26) Jackson, J. D. Classical Electrodynamics; Wiley: New York, 1975; p 39.
(27) Wallqvist, A. Chem. Phys. 1990, 148, 439-449.

(28) Dinur, U. J. Phys. Chem. 1990, 94, 5669-5671.

(29) Dinur, U.; Hagler, A. T. J. Chem. Phys. 1992, 97, 9161-9172.

(30) Mannfors, B.; Mirkin, N. G.; Palmo, K.; Krimm, S. J. Phys. Chem. A 2003, 107, 1825-1832.

(31) Halgren, T. A.; Damm, W. Curr. Opin. Struct. Biol. 2001, 11, 236-242.

(32) Dinur, U. J. Phys. Chem. 1991, 95, 6201-6211.

(33) Palmo, K.; Krimm, S. J. Chem. Theory Comput. 2007, 3, 2120-2127.

(34) Qian, W.; Krimm, S. J. Mol. Struct. (THEOCHEM) 2006, 766, 93-104.

(35) Dykstra, C. E. Acc. Chem. Res. 1988, 21, 355-361.

(36) Andrés, J. L.; Martí, J.; Duran, M.; Lledos, A.; Bertrán, J. J. Chem. Phys. 1991, 95, 3521-3527.

(37) Hermansson, K. J. Chem. Phys. 1993, 99, 861-868.

(38) Zilles, B. A.; Person, W. B. J. Chem. Phys. 1983, 79, 65-77.

(39) Galvez, O.; Gomez, P. C.; Pacios, L. F. J. Chem. Phys. 2001, 115, $11166-11184$.

(40) Spackman, M. A. J. Comput. Chem. 1996, 17, 1-18.

(41) Barnett, R. N.; Landman, U. Phys. Rev. B 1993, 48, 2081-2097.

(42) Gu, Y.; Kar, T.; Scheiner, S. J. Am. Chem. Soc. 1999, 121, 94119422

(43) Lee, H. M.; Suh, S. B.; Lee, J. Y.; Tarakeshwar, P.; Kim, K. S. J. Chem. Phys. 2000, 112, 9759-9772.

(44) Maroulis, G. Chem. Phys. Lett. 1998, 289, 403-411.

(45) Tschumper, G. S.; Leininger, M. L.; Hoffman, B. C.; Valeev, E. F.; Schaefer, H. F., III.; Quack, M. J. Chem. Phys. 2002, 116, 690-701.

(46) Huang, X.; Braams, B. J.; Bowman, J. M.; Kelly, R. E.; Tennyson, J.; Groenenboom, G. C.; van der Avoird, A. J. Chem. Phys. 2008, 128, 034312.

(47) Wang, J.; Zhang, W.; Mukamel, S.; Hochstrasser, R. J. Phys. Chem. B 2008, 112, 5930-5937.

JP806948W 